\title{
Singular Surfaces in Multi-Agent Connectivity Maintenance Games
}

\author{
Sourabh Bhattacharya Tamer Başar Naira Hovakimyan
}

\begin{abstract}
In this paper, we analyze a connectivity maintenance problem that arises when two mobile autonomous agents navigate in an environment containing obstacles. Using the Rician fading model for the communication channel, we address the problem for the case when the motion strategies for one of the agents is adversarial in nature. We investigate a specific kind of singular surface that appears in the solution to the underlying pursuit-evasion game, namely the dispersal surface. We present construction of the projection of several dispersal surfaces for various obstacle geometries by fixing the initial position of the evader. Finally, we present numerical simulations for specific environments containing obstacles.
\end{abstract}

\section{INTRODUCTION}

In the last decade, teams of mobile autonomous agents have been deployed in various military, as well as civilian scenarios, to accomplish tasks in a cooperative manner. Maintaining the connectivity of the underlying communication network in a team seems to be an essential requirement for the convergence and stability of various control laws that have been proposed for accomplishing tasks related to consensus and flocking [18], [16]. A detailed discussion of various connectivity maintenance problems related to multiagent systems can be found in [3]. The limitations on the power of the transmitting antenna sets a maximum range for effective communication among the agents. This restricts the motion of the individual agents since they have to remain connected to the network while avoiding collision with obstacles in the environment. Additionally, the obstacles also deteriorate the quality of communication if they occlude the mutual line-of-sight between two agents. In this paper, we analyze such a scenario that arises when two autonomous mobile agents navigate to explore an environment containing obstacles.

This work is primarily motivated by exploration scenarios involving autonomous agents. The problem of maintaining connectivity also arises in a recent work in [25]. The authors propose the use of mobile robots as network routers which can serve as a cost-effective alternative to the current practice of installing a large number of static routers for the purpose of providing a good coverage for wireless service in indoor environments. In one of the models, the end user is assumed

Sourabh Bhattacharya, Tamer Başar and Naira Hovakimyan are with the Coordinated Science Lab, University of Illinois at Urbana-Champaign, Urbana, IL 61801 \{sbhattac, basar1, nhovakim\}@illinois.edu

This material is based upon work supported in part by the U.S. Army Research Laboratory (ARL) and the U.S. Army Research Office (ARO) under grant number W911NF-09-1-0383 and in part by the U.S. Air Force Office of Scientific Research (AFOSR) under grant number FA9550-09-10249 . to be adversarial in nature resulting in a discrete-time pursuitevasion game. Our work is in the same vein, but with a marked distinction in the analysis. We address the problem in a continuous-time setting, and use the theory of differential games to solve the resulting pursuit-evasion game.

The scenario we address is one of two holonomic agents moving in an environment containing polygonal obstacles. One of the agents, called the pursuer, is assumed to 'follow' the other agent so as to maintain a constant line-of-sight, which is the path of the dominant signal. The other agent is modeled as an adversary that tries to break the line-ofsight with pursuer. Therefore, the problem of maintaining a healthy communication link has been modeled as a visibility based pursuit-evasion game [11].

The theory of deterministic pursuit-evasion games was single-handedly created by Rufus Isaacs, which culminated in his book [10]. An exhaustive analysis of solved and partly solved zero-sum differential games has been provided in [1] and [13]. Most of the classical problems in pursuit-evasion deal with players in obstacle-free space having either constraints on their motion or constraints on their control due to under-actuation. In the recent past, researchers have analyzed pursuit-evasion problems with constraints in the state space. In [15], a pursuit-evasion game has been analyzed with the pursuer and the evader constrained to move on a twodimensional conical surface in a three-dimensional space. A theoretical framework based on the method of characteristics has been presented in [14] to address such problems. The inherent hardness in obtaining an analytical solution to the associated Hamilton-Jacobi-Isaacs equation has led to the development of numerical techniques for the computation of the value function. Recent efforts in this direction to compute an approximation of the reachable sets have been provided by Mitchell and Tomlin [17], Stipanović, Hwang and Tomlin [22], and Stipanović, Sriram and Tomlin [24]. Solutions for particular multi-player games were presented by Pashkov and Terekhov [19], Levchenkov and Pashkov [12], Hagedorn and Breakwell [9], Breakwell and Hagedorn [7], Sriram et al. [21], and recently, by Fuchs et al. [8]. More general treatments of multiplayer differential games were presented by Starr and Ho [6], Vaisbord and Zhukovskiy [26], Zhukovskiy and Salukvadze [27], and Stipanović, Hovakimyan and Melikyan [23].

In contradistinction with our previous work in [3], [2], where a jammer is present in the vicinity, this work deals with establishing a healthy communication link between two agents in the absence of explicit information exchange regarding their motion strategies. An adversarial nature for the motion of one of the agents is assumed in order to foresee 
a worst-case scenario. Section II presents the formal problem statement. Section III summarizes a previous analysis published by the authors to compute the regular trajectories. Section IV presents the computation of dispersal surfaces for the case of two singular points. Section V discusses the computation of the singular surfaces for two corners in the vicinity of other obstacles and generalizes the construction to polygonal environments. Finally, Section VI presents our conclusion and directions for future work.

\section{Problem Formulation}

It is a well-known fact in the communications literature that the signal received at an antenna from a transmitter contains a dominant part, which travels along the direct line-of-sight between the receiver and the transmitter. This phenomenon is captured by the the Rician fading model [20], given by the probability distribution function

$$
p(r)=\left\{\begin{array}{cc}
\frac{r}{\sigma^{2}} e^{-\frac{A^{2}+r^{2}}{2 \sigma^{2}}} I_{0}\left(\frac{A r}{\sigma^{2}}\right) & \text { for } A \geq 0, r \geq 0 \\
0 & \text { for } r<0
\end{array},\right.
$$

where $A$ denotes the peak amplitude of the dominant signal, and $I_{0}(\cdot)$ is the modified Bessel function of the first kind and zero-order. This distribution models the received signal envelope voltage and takes into account a dominant signal path, generally assumed to be the line-of-sight, in addition to weaker multipath signals arriving due to reflections from the environment. Therefore, in order to maintain a healthy communication link, the communicating agents must coordinate their movement so as to maintain a line-of-sight at all times. A plausible way to achieve this is to communicate their control inputs at every instant. This increases the communication network traffic which might lead to congestion. In this work, we explore a non-cooperative strategy to avoid such an unforeseen event.

We consider two agents moving in a planar environment containing obstacles. One of them is assumed to be the pursuer, who tries to keep the other agent, an evader, in its line-of-sight. As previously mentioned, the main motivation behind modelling the other agent as an adversary is to foresee worst-case scenarios that may arise in the future due to for example the agents lacking the information to be able to coordinate their motion effectively. With the aforementioned assumptions, we can formulate the following problem.

Consider a pursuer $(P)$ and an evader $(E)$ that move on a plane with coordinates $\mathbf{x}_{p}=\left(x_{p}, y_{p}\right)$ and $\mathbf{x}_{e}=\left(x_{e}, y_{e}\right)$, respectively. The controls of the pursuer and the evader are denoted as $\hat{v}_{p}=\left(v_{p}, \theta_{p}\right)$ and $\hat{v}_{e}=\left(v_{e}, \theta_{e}\right)$, respectively. where $v_{p}$ and $v_{e}$ are their speeds, and $\theta_{p}$ and $\theta_{e}$ are the corresponding directions of their instantaneous velocities. The maximum speeds of $P$ and $E$ are $\bar{v}_{p}$ and $\bar{v}_{e}$, respectively. The motions of the players are governed by the following equations:

$$
\begin{aligned}
\text { Pursuer : } \dot{x}_{p}=v_{p} \cos \theta_{p}, & \dot{y}_{p}=v_{p} \sin \theta_{p} \\
\text { Evader : } \dot{x}_{e}=v_{e} \cos \theta_{e}, & \dot{y}_{e}=v_{e} \sin \theta_{e}
\end{aligned}
$$

Let $\mathbf{x}=\left[\begin{array}{llll}x_{p} & y_{p} & x_{e} & y_{e}\end{array}\right]^{\prime}$ denote the overall state of the players. The environment contains polygonal obstacles. $W_{\text {free }}$ denotes the free workspace, i.e., the set of all points on the plane that lie outside the obstacles. The visibility polygon of a point $p$ in the workspace, $V(p)$, is the set of points in $W_{\text {free }}$ from which a line segment from $p$ to that point does not intersect the obstacle region. It is assumed that the evader lies in the visibility polygon of the pursuer at the beginning of the game.

The pursuer assumes that the evader is antagonistic in nature, and wants to escape from its visibility in the least amount of time possible. To prevent the evader from escaping, the pursuer tries to keep the evader in its visibility polygon, $V(\mathbf{p}(t))$, for the maximum amount of time. The game ends when the evader breaks the line-of-sight with the pursuer around any vertex of the polygonal obstacles. Let $T$ denote the time instant at which the evader succeeds in breaking the line-of-sight. The outcome of the game is given by the following function

$$
\pi\left(\hat{v}_{p}(\cdot), \hat{v}_{e}(\cdot)\right)=\int_{0}^{T} d t=T
$$

As stated earlier, the objective of the pursuer is to maximize this objective function, which is the time elapsed. On the other hand, the objective of the evader is to minimize it.

\section{Regular AnALYSis}

In this section, we summarize our previous results from [4] and [5]. Let $J(\mathbf{x})$ denote the value of the game at a state $\mathbf{x}$, which we assume to be $C^{2}(\mathbf{x})$. The Hamiltonian of the system is given by the following expression:

$$
\begin{array}{r}
H\left(\mathbf{x}, J_{\mathbf{x}}\right)=1+J_{x_{p}} v_{p} \cos \theta_{p}+J_{y_{p}} v_{p} \sin \theta_{p}+ \\
J_{x_{e}} v_{e} \cos \theta_{e}+J_{y_{e}} v_{e} \sin \theta_{e}
\end{array}
$$

Using Isaacs' first condition from [1] and [10], we obtain the following expressions for the optimal controls of the players

$$
\begin{aligned}
&\left(v_{p}^{*}, v_{e}^{*}, \theta_{p}^{*}, \theta_{e}^{*}\right)=\arg \max _{v_{p}, \theta_{p} v_{e}, \theta_{e}} H\left(\mathbf{x}, J_{\mathbf{x}}\right) \\
& \Rightarrow v_{p}^{*}=\bar{v}_{p}, \quad\left(\cos \theta_{p}^{*}, \sin \theta_{p}^{*}\right) \|\left(J_{x_{p}}, J_{y_{p}}\right) \\
& v_{e}^{*}=\bar{v}_{e}, \quad\left(\cos \theta_{e}^{*}, \sin \theta_{e}^{*}\right) \|\left(-J_{x_{e}},-J_{y_{e}}\right),
\end{aligned}
$$

where || implies that the vectors are mutually parallel. Additionally, $J(\mathbf{x})$ satisfies the Hamilton-Jacobi-Isaacs equation

$$
\begin{gathered}
\frac{\partial J_{\mathbf{x}}}{\partial \tau}-\frac{\partial H\left(\mathbf{x}, J_{\mathbf{x}}\right)}{\partial \mathbf{x}}=0 \\
\Rightarrow J_{\mathbf{x}}=J_{\mathbf{x}}^{0}
\end{gathered}
$$

In the above equation, $\tau$ is the retrograde time, i.e., the time remaining from termination. Substituting (4) into (3) leads to the following relation between the optimal controls and the value functions at terminal conditions:

$$
\begin{gathered}
v_{p}^{*}=\bar{v}_{p}, \quad\left(\cos \theta_{p}^{*}, \sin \theta_{p}^{*}\right) \|\left(J_{x_{p}}^{0}, J_{y_{p}}^{0}\right) \\
v_{e}^{*}=\bar{v}_{e}, \quad\left(\cos \theta_{e}^{*}, \sin \theta_{e}^{*}\right) \|\left(-J_{x_{e}}^{0},-J_{y_{e}}^{0}\right)
\end{gathered}
$$



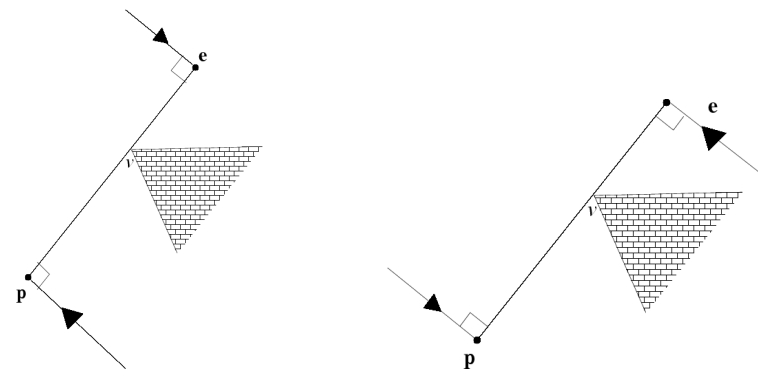

Fig. 1. Optimal trajectories to a termination situation

Let $\left(x_{c}, y_{c}\right)$ be the coordinates of the corner around which the game terminates. The terminal situations around the corner are characterized by the following equation

$$
\frac{\sqrt{\left(y_{e}-y_{c}\right)^{2}+\left(x_{e}-x_{c}\right)^{2}}}{\sqrt{\left(y_{p}-y_{c}\right)^{2}+\left(x_{p}-x_{c}\right)^{2}}} \leq \frac{\bar{v}_{e}}{\bar{v}_{p}}=a
$$

An equality sign between the two terms on the left represents the manifold that forms the boundary of the termination situations locally around the corner. We can characterize the terminal manifold using the variables $\left(x_{p}, x_{e}, y_{e}\right)$. Since $J \equiv 0$ at termination, we obtain the following relations.

$$
\begin{gathered}
J_{x_{p}}^{0}+J_{y_{e}}^{0} \frac{\partial y_{p}}{\partial x_{p}}=0, \quad J_{x_{e}}^{0}+J_{y_{e}}^{0} \frac{\partial y_{p}}{\partial x_{e}}=0 \\
J_{y_{e}}^{0}+J_{y_{e}}^{0} \frac{\partial y_{p}}{\partial y_{e}}=0
\end{gathered}
$$

From (6) and (7), we obtain the following expression for $J_{y_{p}}^{0}$ :

$$
\left|J_{y_{p}}^{0}\right|=\frac{1}{\left(\sqrt{\frac{s_{2}^{2}}{s_{1}^{2}}+1}\right)\left(\bar{v}_{e} \sqrt{\frac{s_{3}^{2}}{s_{1}^{2}}}-\bar{v}_{p}\right)}
$$

where,

$$
\begin{aligned}
& s_{1}=x_{e}-x^{c} \\
& s_{2}=y_{e}-y^{c} \\
& s_{3}=x_{p}-x^{c}
\end{aligned}
$$

Depending on the sign of $J_{y_{p}}^{0}$, there are two possible trajectories for the players before termination. This is shown in Figure 1. The trajectory of the players shown in the figure on the right does not correspond to a saddle-point strategy. Therefore, the trajectory of the players on the left is the only possible optimal strategy before termination. From the analysis, we conclude that the players move along straight lines in opposite directions, orthogonal to the line-of-sight at termination.

Dispersal surfaces are commonly encountered in games of degree. These are singular surfaces on which the players have more than one saddle-point strategy that leads to the same payoff at termination [1], [10], [13]. In the following sections, we present the construction of dispersal surfaces.

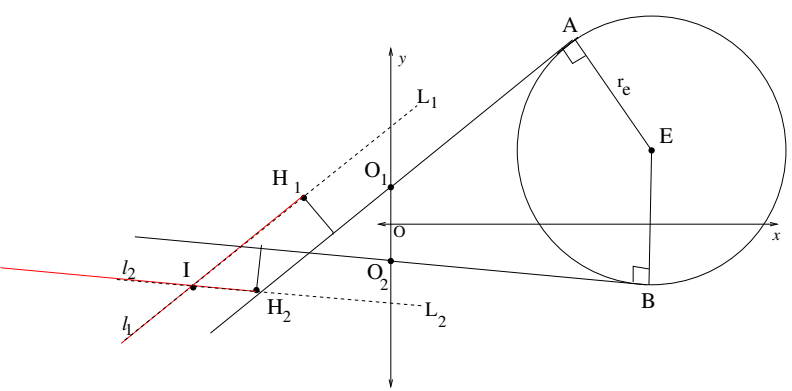

Fig. 2. Geometry around two point obstacles

\section{TWO SINGULAR POINTS}

In this section, we present the construction of a dispersal surface due to the presence of two point obstacles in the workspace. Since the state space lies in $\mathbb{R}^{4}$, the dispersal surfaces lie on a three-dimensional manifold. In order to depict them on a two-dimensional workspace, we fix the initial position of the evader and compute the set of initial positions of the pursuer that lead the players to lie on a dispersal surface.

Referring to Figure 2, let $O_{1}=(0, a)$ and $O_{2}=(0,-a)$ denote the positions of the two point obstacles. Let $E \equiv$ $\left(x_{e}, y_{e}\right)$ denote the initial position of the evader. Let $t$ denote the time of termination of the game, i.e., if the evader is initially at $E$, it breaks the line-of-sight with the pursuer for the first time at time $t$.

Since the maximum speed of the evader is $\bar{v}_{e}$, the reachable set of the evader at time $t$ is $B_{\bar{v}_{e} t}[E]$, where $B_{r}[x]=$ $\left\{y \in \mathbb{R}^{2} \mid\|x-y\| \leq r\right\}$. Let $\mathcal{D}$ denote $B_{\bar{v}_{e} t}[E]$. An infinite number of trajectories for the evader are possible that lie inside $\mathcal{D}$ and do not violate the constraints on the maximum speed of the evader. Nevertheless, we are only interested in trajectories traced by the evader when it follows its saddlepoint strategies, which are given by the following theorem. Refer to Figure 2.

Theorem 1: At termination, the evader is either at $A$ or $B$ which are the points at which tangents from corners $O_{1}$ and $\mathrm{O}_{2}$ meet $\mathcal{D}$, respectively.

Proof: Let us consider the case when the evader breaks the line-of-sight with the pursuer around $O_{1}$. As summarized in Section III, it was shown in [5] that the optimal strategy for the evader is to move on a straight line with speed $\bar{v}_{e}$ before termination. Therefore, the evader must lie on $\partial \mathcal{D}$ (boundary of $\mathcal{D}$ ) at termination. Moreover, the evader's path must be perpendicular to the line joing $O_{1}$ and the instantaneous position of the evader at termination. Figure 3 shows the two possible positions of the evader on $\partial \mathcal{D}$ that satisfy the aforementioned conditions at termination: $A$ and $A^{\prime}$. The line segments $\overline{O_{1} A}$ and $\overline{O_{1} A^{\prime}}$ are tangent to $\partial \mathcal{D}$. Using arguments similar to the ones that eliminate one of the possible trajectories of the players before termination in Section III, we can conclude that $A$ is the only possible position of the evader at termination. Similarly, $B$ is the only position of the evader at termination if the evader breaks the line-of-sight around $\mathrm{O}_{2}$, as shown in Figure 2. 


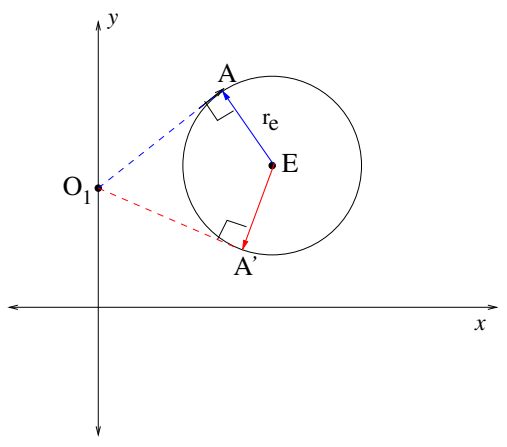

Fig. 3. Two possible positions of the evader at termination

Next, we characterize the initial positions of the pursuer from which the evader can terminate the game in $t$ units of time. Refer to Figure 4. Consider the case when termination occurs around $O_{1}$. From (6), we know that the pursuer lies on $\overrightarrow{D_{1} F}$ at termination. Moreover, the optimal strategy of the pursuer is to follow a straight line, perpendicular to $\overrightarrow{A F}$. From the above facts, we can conclude that the set of all initial positions of the pursuer is given by the ray $l_{1}$. Similarly, if the termination occurs around the corner $\mathrm{O}_{2}$, the initial position of the pursuer lies on ray $l_{2}$. Using geometric arguments, it can be easily shown that the lines on which the rays $l_{1}$ and $l_{2}$ lie, is given by the following

$$
\begin{gathered}
y=\overbrace{\left[\frac{\left(y_{e} \pm a\right) \sqrt{x_{e}^{2}+\left(y_{e} \pm a\right)^{2}-\bar{v}_{e}^{2} t^{2}} \mp x_{e} \bar{v}_{e} t}{x_{e} \sqrt{x_{e}^{2}+\left(y_{e} \pm a\right)^{2}-\bar{v}_{e}^{2} t^{2}} \pm\left(y_{e} \pm a\right) \bar{v}_{e} t}\right]}^{m_{ \pm}} \mp \\
\underbrace{a \mp \bar{v}_{p} t \sqrt{1+m_{2}^{2}}}_{c_{ \pm}},
\end{gathered}
$$

where the pair $\left(m_{+}, c_{+}\right)$and $\left(m_{+}, c_{+}\right)$are the slopes and y-intercepts of $L_{1}$ and $L_{2}$, respectively.

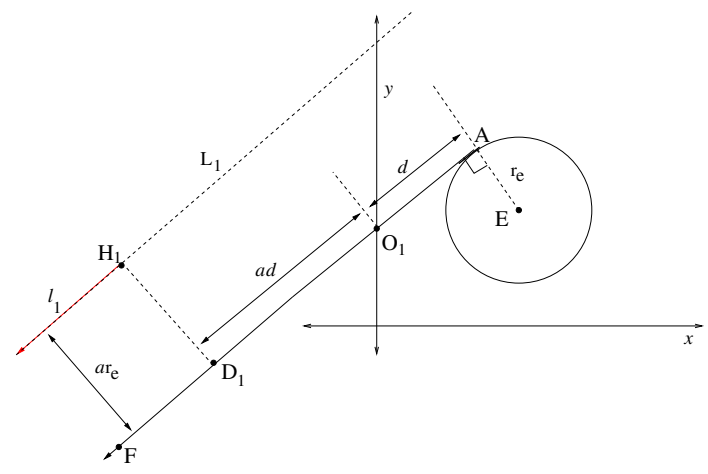

Fig. 4. Positions of the pursuer at the initial and terminal time.

From the above discussions, we can conclude the following. Let $I$ denote the intersection of rays $l_{1}$ and $l_{2}$. If the initial positions of the pursuer and the evader are $I$ and $E$, respectively, the evader has a choice between breaking the line-of-sight around $\mathrm{O}_{1}$ as well as $\mathrm{O}_{2}$, with both options leading to the same termination time. Therefore, the initial position of the players corresponding to this situation lies
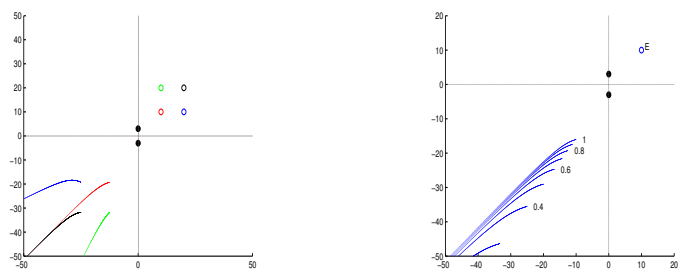

(a) Different evader positions. (b) Different evader speeds.

Fig. 5. Singular surfaces for a point obstacle.

on a dispersal surface. The optimal strategy of the pursuer depends on its knowledge about the instantaneous velocity of the evader. The coordinates of $I$ are given by the following expressions

$$
x_{p}=\frac{c_{-}-c_{+}}{m_{-}-m_{+}} ; \quad y_{p}=\frac{m_{+} c_{-}-m_{-} c_{+}}{m_{+}-m_{-}}
$$

For a fixed initial position of the evader, $I$ traces a curve as $t$ varies. This curve is the projection of the three-dimensional dispersal surface along the initial position of the evader.

Figure 5 illustrates the dispersal surfaces for two different scenarios. The positions of the point obstacles are $(0,3)$ and $(0,-3)$. The maximum speed of the pursuer is assumed to be 1. In Figure 5(a), the dispersal surfaces are shown for four different initial positions of the evader. In Figure 5(b), dispersal surfaces are shown for varying maximum speeds of the evader.

\section{TWO CORNERS IN A GENERAL POLYGONAL ENVIRONMENT}

In this section, we extend the results of the previous section and present an explicit construction of the projection of the dispersal surfaces that arise due to the intersection of the optimal paths emanating backwards in retrograde time from two corners, in the presence of other obstacles.

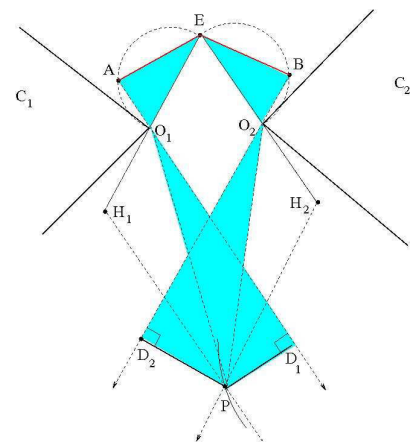

Fig. 6. Dispersal surface in the presence of a corner

Referring to Figure 6, consider an environment having polygonal obstacles. Let $E=\left(x_{e}, y_{e}\right)$ denote the initial position of the evader on the plane. Let $O_{1}$ and $O_{2}$ be corners of obstacles $C_{1}$ and $C_{2}$, respectively, not necessarily distinct, and visible from $E$. Similar to the notation adopted in the previous section, let $A$ and $B$ denote the position of the evader at termination when it breaks the line-of-sight with 


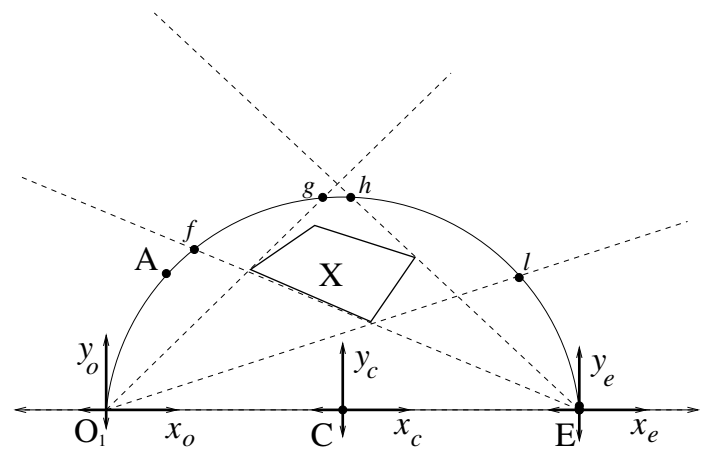

Fig. 7. Construction of $\tilde{S}_{1}$

the pursuer around $O_{1}$ and $O_{2}$, respectively. Since $A$ is the position of the evader at the termination of a play, it must satisfy the following conditions:

1) $A \in W_{\text {free }}$

2) $A$ lies on the arc of the semi-circle with $O_{1} E$ as the diameter.

3) $O_{1}$ is visible to the evader as it moves on a straight line joining $E$ and $A \Longrightarrow A E \in V\left(O_{1}\right)$.

Similar conditions hold for $B$ to qualify as a terminating position for the evader around corner $\mathrm{O}_{2}$.

Let $\tilde{S}_{1}$ and $\tilde{S}_{2}$ denote the set of all points that satisfy the above conditions around corners $O_{1}$ and $O_{2}$, respectively. Now we present the construction of $\tilde{S}_{1}$. Referring to Figure 7 , let $C$ denote the center of the semi-circular arc $O_{1} A E$. We attach a local coordinate frame to each of the points $C, O_{1}$ and $E$, as shown in the figure. Angles are measured counter-clockwise with respect to the $x$-axis of the frame in context. $X$ denotes an obstacle inside the closure of the semi-circular disk $O_{1} A E$. Let $\partial X$ denote the boundary of the obstacle. Since we assume that the evader can see the pursuer initially, $E$ does not qualify as a point at which the evader can terminate the game. Moreover, we assume that an obstacle is a closed polygonal set. Therefore, the terminal position of the evader can lie on the corner $O_{1}$ since it is not in $W_{\text {free. }}$. Let $\tilde{K}$ denote the set of points on the arc $O_{1} A E$ excluding the points $O_{1}$ and $E$. There exists a natural bijective map $\gamma: \tilde{K} \rightarrow(0, \pi)$ that maps any point in $\tilde{K}$ to its radial angle $\theta_{c}$ in the coordinate frame attached to $C$. Let $S \subseteq \tilde{K}$ denote the set of all points that are either occluded from $O_{1}$ or $E$ by $X$. Let the tangents from $E$ to $X$ intersect $\tilde{K}$ at $f$ and $h$. Without loss of generality, let $\theta_{c}(f)>\theta_{c}(h)$. Similarly, the tangents from $O_{1}$ to $X$ intersect $\tilde{K}$ at $g$ and $i$ with $\theta_{c}(g)>\theta_{c}(i) \cdot \gamma(S)$ is a closed interval in $(0, \pi)$. Even if the obstacles have a non empty intersection with $\tilde{K}$, the previous statement remains true.

Now let us consider the case when there are $n>1$ obstacles in the closure of the semi-circular disk. For each obstacle $X_{i}$, we repeat the following steps to construct the corresponding set $S^{i}$.

1) Construct the tangents from $O_{1}$ to obstacle $i$.

2) Compute the intersection of the tangents with $\tilde{K}$. Let the points be denoted as $g$ and $l$ with $\theta_{c}(g)>\theta_{c}(l)$.
3) Compute the intersection of the tangents from $E$ to $\tilde{K}$ and denote the points as $f$ and $h$ with $\theta_{c}(f)>\theta_{c}(h)$. $S^{i}$ contains the the set of points $p \in \tilde{K}$ such that $\theta_{c}(l) \leq$ $\theta_{c}(p) \leq \theta_{c}(f)$. In other words, $S^{i}$ consists of the set of points that are either occluded from $O_{1}$ or $E$ by $X_{i}$. Define $S_{1}=\tilde{K} \backslash \cup_{i=1}^{n} S^{i}$. Since $S_{1}$ is a union of open intervals, it is open. Therefore $\gamma\left(S_{1}\right)=\bigcup_{i=1}^{k_{1}}\left(\theta_{i}, \theta_{i+1}\right)$, where $\theta_{i}=\theta_{c}(l)$ corresponding to obstacle $X_{i}$. Every point in $S_{1}$ is associated with a unique termination time that is proportional to the distance of that point from $E$. Therefore, we can define a bijective map $\mathcal{I}_{1}: S_{1} \rightarrow R$, such that $\mathcal{I}_{1}(p)=t_{p}$ where $t_{p}$ is the time of termination of the game if the evader starts at $E$. From the definition of $S_{1}$ and the fact that it is the union of open intervals, we can conclude that $\mathcal{I}_{1}\left(S_{1}\right)=$ $\bigcup_{i=1}^{k_{1}}\left(t_{i}, t_{i+1}\right)$. Since $\mathcal{I}_{1}$ is bijective, $\mathcal{I}_{1}\left(S_{1}\right) \simeq S_{1}$. Similarly, we can define sets $S_{2}$ and $I_{2}$ for termination around $O_{2}$.

Every point $q \in S_{1}$ has an associated time of termination $t_{q}$. The termination positions of the evader for which $E$ lies on a dispersal surface are given by the following sets:

$$
\begin{array}{lll}
\tilde{S}_{1}=\left\{q \in S_{1} \mid \exists q^{\prime} \in S_{2}\right. & \text { s.t. } & t_{q}=t_{q^{\prime}} \\
\tilde{S}_{2}=\left\{q \in S_{2} \mid \exists q^{\prime} \in S_{1}\right. & \text { s.t. } & t_{q}=t_{q^{\prime}}
\end{array}
$$

From the above, if the players start from an initial position that lies on a dispersal surface, then $\tilde{S}_{1}$ and $\tilde{S}_{2}$ are the maximal subsets of $S_{1}$ and $S_{2}$, respectively, comprising of terminal positions of the evader, starting at $E$.

Lemma 1: The set of points in $\tilde{S}_{1}$ and $\tilde{S}_{2}$ is a union of open intervals of the form $\left(q_{1}, q_{2}\right)$, where $q_{1}, q_{2} \in S_{1}$.

Proof: By definition, $\mathcal{I}_{1}\left(S_{1}\right)=\bigcup_{i=1}^{k_{1}}\left(t_{i}, t_{i+1}\right)$ and $\mathcal{I}_{2}\left(S_{2}\right)=\bigcup_{i=1}^{k_{2}}\left(t_{i}, t_{i+1}\right)$. Therefore, $T=\mathcal{I}_{1}\left(S_{1}\right) \cap \mathcal{I}_{2}\left(S_{2}\right)$ is open, since it is an intersection of finite number of open sets. Moreover, it is also a union of open intervals. Since $\mathcal{I}_{1}$ and $\mathcal{I}_{2}$ are bijective, $\tilde{S}_{1}=\mathcal{I}_{1}^{-1}(T)$ and $\tilde{S}_{2}=\mathcal{I}_{2}^{-1}(T)$ is a union of open intervals.

Let $\tilde{P}$ contain the initial positions of the pursuer that lie on the dispersal surface when the evader is initially at $E$. Now we present the construction of $\tilde{P}$ from the sets $\tilde{S}_{1}$ and $\tilde{S}_{2}$. Let $q_{1} \in \tilde{S}_{1}$ and $q_{2} \in \tilde{S}_{2}$ such that $t_{q_{1}}=t_{q_{2}}$. The intersection of the lines parallel to $q_{1} \mathrm{O}_{1}$ from $\mathrm{H}_{1}$ and $q_{2} \mathrm{O}_{2}$ from $H_{2}$ gives the point $p$. For $p$ to lie in $\tilde{P}$, it should satisfy the following conditions:

1) $p, D_{1}, D_{2} \in W_{\text {free }}$

2) $p \in V\left(D_{1}\right) \cap V\left(D_{2}\right)$

3) $\left(1-t^{\prime}\right) p+t^{\prime} D_{1} \in V\left(t^{\prime} E+\left(1-t^{\prime}\right) A\right)$ and $\left(1-t^{\prime}\right) p+$ $t^{\prime} D_{2} \in V\left(t^{\prime} E+\left(1-t^{\prime}\right) B\right) \quad \forall t^{\prime} \in[0, t]$

The third condition ensures that the pursuer and the evader are visible to each other at all times $t^{\prime} \leq t$. For all points $p \in \tilde{P}$, we can obtain the coordinates $\left(x_{p}, y_{p}\right)$ using (10).

We can extend the above analysis to any general environment containing polygonal obstacles. For any two corners $\mathrm{O}_{1}$ and $\mathrm{O}_{2}$, first compute the dispersal surface from the algorithm presented in the aforementioned analysis. Next, consider all possible pairs of corners $O_{1}$ and $O_{2}$ and compute the dispersal surface for each pair. This completes the construction of the dispersal surface for a given initial position of the evader $E=\left(x_{e}, y_{e}\right)$ in a general polygonal environment. 


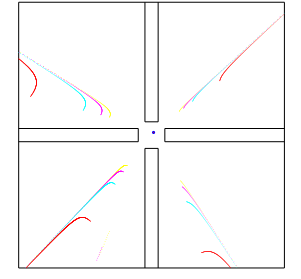

(a) (b)

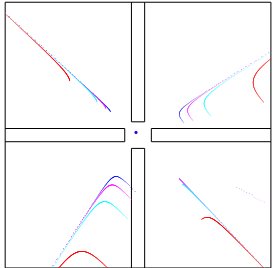

Fig. 8. Projection of the dispersal surface for two different initial positions of the evader in a closed environment containing obstacles. The center of the outermost square is the origin. In figure (a), the evader is initially at $(0.3,0.4)$. In figure (b), the evader is initially at $(-0.3,0.5)$. The different colors correspond to dispersal surfaces for different values of $a$. The color blue denotes the case, $a=1$. The color magenta denotes the case, $a=0.75$. The color cyan denotes the case $a=0.5$. The color red denotes the case, $a=0.25$.

Figure 8 shows the projection of the dispersal surfaces on the plane for an initial position of the evader. We can see that as the speed of the evader increases, the dispersal surfaces get closer to the corners of the environment. This is intuitive since with increasing speed, the evader can start from a position that is closer to the corner in order to break the line of sight.

\section{CONCLUSION}

In this paper, we analyzed a connectivity maintenance problem that arises when two mobile agents navigate in an environment containing obstacles. Using the Rician fading model for the communication channel and an adversarial model for the receiver, we reduced the problem to a visibility-based pursuit-evasion game. We investigated a specific kind of a singular surface, called the dispersal surface, which appears in the solution to the pursuit-evasion problem. We presented the construction of the projection of dispersal surfaces in the workspace and conducted simulations it for specific environments.

One of the future directions is to explore the existence of other kinds of singular surfaces in the above game. Additionally, different versions of the game can be considered in which the players have constraints in sensing and locomotion. Another direction of future research would be to develop numerical techniques for computing the value function that yields the optimal trajectories.

\section{REFERENCES}

[1] T. Başar and G. J. Olsder. Dynamic Noncooperative Game Theory. SIAM, Philadelphia, PA, second edition, 1999.

[2] S Bhattacharya and T. Başar. Game-theoretic analysis of an aerial jamming attack on a UAV communication network. In Proceedings of American Control Conference, pages 818-823, Baltimore, Maryland, June 2010.

[3] S. Bhattacharya and T. Başar. Graph-theoretic approach to connectivity maintenance in mobile networks in the presence of a jammer. In IEEE Conference on Decision and Control, pages 3560-3565, Atlanta, GA, December 2010.

[4] S. Bhattacharya and S. Hutchinson. On the existence of Nash equilibrium for a two-player pursuit-evasion game with visibility constraints. International Journal of Robotics Research, 29(7):831839, 2010.
[5] S. Bhattacharya, S. Hutchinson, and T. Başar. Game theoretic analysis of pursuit-evasion game with visibility constraints. In American Control Conference, pages 373-378, 2009.

[6] J. V. Breakwell and P. Hagedorn. Further properties of non-zereo sum differential games. Journal of Optimization Theory and Applications, 3:207-219, 1969.

[7] J. V. Breakwell and P. Hagedorn. Point capture of two evaders in succession. Journal of Optimization Theory and Applications, 27:8997, 1979.

[8] Z Fuchs, P Khargonekar, and J. Evers. Cooperative defense within a single-pursuer, two-evader pursuit evasion differential game. In Proceedings of Conference on Decision and Control, pages 30913097, Atlanta, Georgia, December 2010.

[9] P. Hagedorn and J. V. Breakwell. A differential game of approach with two pursuers and one evader. Journal of Optimization Theory and Applications, 18:15-29, 1976.

[10] R. Isaacs. Differential Games. Wiley, New York, NY, 1965.

[11] S. M. LaValle. Algorithms for Planning. The MIT Press, Cambridge, MA, 2005.

[12] A. Y. Levchenkov and A. G. Pashkov. Differential game of optimal approach of two inertial pursuers to a noninertial evader. Journal of Optimization Theory and Applications, 65:501-518, June 1990.

[13] J. Lewin. Differential Games: Theory and Methods for Solving Game Problems with Singular Surfaces. Springer-Verlag, London, England, 1994.

[14] A. A. Melikyan. Generalized Characteristics of First Order PDEs: Applications in Optimal Control and Differential Games. Birkhauser, Boston, MA, 2000.

[15] A. A. Melikyan and N. V. Hovakimyan. Game problem of simple pursuit on a two-dimensional cone. Journal of Applied Mathematics and Mechanics, 55(5):607-618, 1991.

[16] M. Mesbahi and M. Egerstedt. Graph Theoretic Methods in Multiagent Networks. Princeton University Press, New Jersey, 2010.

[17] I. M. Mitchell and C. J. Tomlin. Overapproximating reachable sets by Hamilton-Jacobi projections. Journal of Scientific Computing, 19:323346, 2003.

[18] R. Olfati-Saber and R. M. Murray. Consensus problems in networks of agents with switching topology and time delay. IEEE Transactions on Automatic Control, 49(9):1520-1533, 2004.

[19] A. G. Pashkov and S. D. Terekhov. A differential game of approach with two pursuers and one evader. Journal of Optimization Theory and Applications, 55:303-311, July 1987.

[20] T. S. Rappaport. Wireless Communications: Principles and Practice, 2nd Ed. Prentice Hall, New Jersey, 2002.

[21] S. Shankaran, D. Stipanović, and C. Tomlin. Collision avoidance strategies for a three player game. Annals of International Society of Dynamic Games, 2010. To appear.

[22] D. M. Stipanović, I. Hwang, and C. J. Tomlin. Computation of an over-approximation of the backward reachable set using subsystem level set functions. Dynamics of Continuous, Discrete and Impulsive Systems, 11:399-411, 2004.

[23] D. M. Stipanović, A. A. Melikyan, and N. V. Hovakimyan. Some sufficient conditions for multi-player pursuit evasion games with continuous and discrete observations. Annals of the International Society of Dynamic Games, 10:133-145, 2009.

[24] D. M. Stipanović, S. Shankaran, and C. Tomlin. Strategies for agents in multi-player pursuit-evasion games. In Eleventh International Symposium on Dynamic Games and Applications, Tucson, AZ, 2006.

[25] O. Tekdas, W. Yang, and V. Isler. Robotic routers: Algorithms and implementation. Int. Journal of Robotics Research, 29(1), 2010.

[26] E. M. Vaisbord and V. I. Zhukovskiy. Introduction to Multi-Player Differential Games and their Applications. Gordon and Breach, New York, NY, 1988.

[27] V. I. Zhukovskiy and M. E. Salukvadze. The Vector Valued Maxmin. Academic Press, San Diego, CA, 1994. 